\title{
Treatment decisions, clinical outcomes, and pharmacoeconomics in the treatment of patients with EGFR mutated stage III/IV NSCLC in Germany: an observational study
}

Wolfgang Schuette ${ }^{1 *}$, Peter Schirmacher ${ }^{2}$, Wilfried E. E. Eberhardt ${ }^{3}$, Manfred Dietel ${ }^{4}$, Ute Zirrgiebel ${ }^{5}$, Lars Muehlenhoff ${ }^{6}$ and Michael Thomas ${ }^{7}$

\begin{abstract}
Background: We evaluated treatment decisions and outcomes in a cohort of predominately Caucasian patients with EGFR mutation-positive (EGFR Mut+) non-small-cell lung cancer (NSCLC).

Methods: REASON (NCT00997230) was a non-interventional study in German patients with stage IIIB/IV NSCLC. Secondary endpoints for EGFR Mut + NSCLC included progression-free survival (PFS), overall survival (OS), adverse event (AE) management, and pharmacoeconomic outcomes.
\end{abstract}

Results: Among 334 patients with EGFR Mut + NSCLC, tyrosine kinase inhibitors (TKIs) were the most common first-line therapy (56.6\%, 53.0\% gefitinib). Among patients who received TKIs/gefitinib before first disease progression, PFS was longer compared with those who did not receive a TKI (median 10.1/10.0 vs. 7.0 months; HR 0.67/0.69; log-rank $p=0.012$ / $p=0.022$ ). OS was longer for those patients who ever received a TKl/gefitinib during their complete therapy course compared with those who never received a TKI (median 18.4/18.1 vs. 13.6 months; HR 0.53/0.55; $p=0.003 / p=0.005$ ). Total mean first-line treatment healthcare costs per person were higher for those receiving TKIs $(€ 46,443)$ compared with those who received chemotherapy $(€ 27,182)$. Mean outpatient and inpatient costs were highest with chemotherapy. Rash, diarrhea, and dry skin were the most commonly reported AEs for patients receiving gefitinib.

Conclusions: In REASON, TKI therapy was the most common first- and second-line treatment for EGFR Mut + NSCLC, associated with increased drug costs compared with chemotherapy. Patients who received gefitinib or a TKI ever during their complete therapy course had prolonged PFS and OS compared with patients who did not receive a TKl.

Trial registration: The trial was registered on October, 2009 with ClinicalTrials.gov: https://linicaltrials.gov/ct2/show/ NCT00997230?term=NCT00997230\&rank=1

Keywords: EGFR-mutations, Non-small cell lung cancer (NSCLC), EGFR tyrosine kinase inhibitor, Observational, REASON study

\footnotetext{
* Correspondence: Wolfgang.Schuette@Martha-Maria.de;

Studiensekretariat.Schuette@nicsys.de

${ }^{1}$ Krankenhaus Martha-Maria Halle-Doelau gGmbH, Klinik für Innere Medizin II,

Roentgenstr, 106120 Halle, Germany

Full list of author information is available at the end of the article
} 


\section{Background}

Non-small cell lung cancer (NSCLC) accounts for 85-90\% of lung cancers [1]. Among those patients with NSCLC, mutations in the epidermal growth factor receptor (EGFR) are present in $30-40 \%$ of Asian patients and $10-20 \%$ of white patients [2]. EGFR tyrosine kinase inhibitors (TKIs), such as gefitinib have demonstrated efficacy compared with chemotherapy in patients with locally advanced or metastatic NSCLC with activating mutations of the TK domain of the EGFR [3, 4]. EGFR testing is now a standard approach in the work-up of patients with advanced NSCLC and is recommended by the ESMO Clinical Practice European guidelines and German lung cancer guidelines $[1,5]$.

The primary aim of this non-interventional study, Registry for the Epidemiological and Scientific evaluation of EGFR mutation status in patients with newly diagnosed locally advanced or metastatic NSCLC (REASON), was to generate data on EGFR mutation status from a large cohort of predominantly Caucasian patients and to correlate it with clinicopathological characteristics. Detailed primary endpoint results from REASON are reported in a separate publication [6]. In summary, among 4200 evaluable patients, 431 (10.3\%) had EGFR mutation-positive (Mut+) disease. The odds of EGFR mutation were significantly higher $(P<0.0001)$ in females versus males (odds ratio 1.85; $95 \%$ confidence interval 1.48, 2.32), never smokers versus ever smokers $(3.64 ; 2.91,4.56)$, and adenocarcinoma versus other histological sub-types $(2.94 ; 2.17,4.08)$.

In this paper, we report the results for the secondary endpoints of REASON, including detailed analyses of treatment decisions, clinical outcome, safety and tolerability (restricted to patients with EGFR Mut + NSCLC who received gefitinib), and pharmacoeconomic outcomes. We also report explorative analyses of clinical outcomes in patients with EGFR Mut + NSCLC who received gefitinib, which was the most commonly prescribed first-line EGFR-TKI.

\section{Methods}

The study design has been reported in detail elsewhere [6]. Briefly, this was a national, multicenter, prospective, observational study carried out in 149 centers in Germany in patients with newly diagnosed stage IIIB/IV NSCLC (NCT00997230). Patients were treated and assessed under real-life conditions and data were taken from the electronic case report form.

Given the non-interventional design of the study, intervals for follow-up were conducted according to the routine practice of the centers. Responses were documented according to the radiologist's report (and not according to pre-specified criteria) and could be radiological or clinical, as judged by the investigator. Formal Response Evaluation Criteria In Solid Tumors (RECIST) was not performed.
Patients were $\geq 18$ years with histologically confirmed stage IIIB/IV NSCLC and suitable for first-line treatment, but not amenable to curative surgery or radiotherapy, and with suitable tumor tissue available for EGFR testing [6]. Participation was until documentation of the first-line treatment decision. Patients with EGFR Mut + NSCLC receiving first-line therapy, and not participating in other interventional studies, could continue until patients' decision to withdraw, death, or loss to follow-up.

\section{Endpoints}

The primary endpoint of the study has been reported previously [6]. Secondary endpoints were analyzed only for patients with EGFR Mut + disease who were not participating in other clinical trials, with the exception of first-line treatment decisions and concomitant therapy, which were investigated in all patients.

Treatment decisions were recorded for first-line and planned second-line treatments. Multiple agents could be recorded for treatment decisions. Amendments to the protocol allowed for extended data capture (subject to consent of patients and data cut-off at 31 October 2012): documentation of actual treatments beyond first-line, extension of follow-up until patient's death, and retrospective documentation of the date of death for all patients with EGFR Mut + disease (as assessed by Ethics Committee).

Clinical outcome records included progression-free survival (PFS), overall survival (OS), and response rate (RR) (complete response plus partial response). Disease control rate was originally designated as an endpoint but could not be determined due to the unknown duration of stable disease resulting from the lack of a standardized frequency of follow-up documentation.

Reported adverse events (AEs) for supportive treatments and $\mathrm{AE}$ management associated with first-line treatment in patients receiving gefitinib were recorded. AEs reported more than once for a patient, and with at least one occurrence considered by the physician to be gefitinib related, were classified as adverse drug reactions (ADRs). AEs were graded according to the National Cancer Institute Common Terminology Criteria for Adverse Events Version 3.0.

Resource use and costs were analyzed for first-line drug therapy (based on type and duration of therapy and priced using the LAUER-TAXE ${ }^{\bullet}$ price list, a German price list reflecting the official prices for prescribed pharmaceuticals). Outpatient care costs were based on the number of outpatient visits according to the physicians' specialty and services used, and calculated using the Doctors' Fee Scale within the Statutory Health Insurance Scheme (Einheitlicher 
Bewertungsma stab). Inpatient care costs were based on the number of inpatient stays and the number of days in hospital associated with the event and calculated using the national Diagnosis-Related Groups for inpatient services. Auxiliary nursing support and incapability to work (based on changes between baseline and end of the observation period) were also recorded; however, no costs were assigned to these.

\section{Statistical methods}

Descriptive statistics were used with 95\% confidence limits. Binary, categorical, and ordinal parameters were summarized by means of absolute numbers and percentages (including 'missing data' as a valid category). Statistical tests, which were performed two-sided at a 5\% level of significance, were descriptive-exploratory.

A multivariate logistic regression analysis of factors influencing first-line therapy decisions (TKI vs. no TKI) was conducted including: mutational status known at therapy initiation, age, gender, smoking status, tumor histology, disease status at diagnosis, Eastern Cooperative Oncology Group performance status, tumor stage, and tumor grade (Grade 1 [well differentiated] to Grade X [cannot be assessed]). For clinical outcomes, analysis was performed by receipt of TKI/gefitinib vs. no TKI. The Kaplan-Maiermethod was used to estimate PFS and OS. Patients without an event at data cut-off were censored cases.

For pharmacoeconomic analyses, descriptive statistics for the costs were computed for continuous variables over the observation period. Subgroup analysis was performed according to therapy received (chemotherapy or TKI) in the first-line setting, including those patients who switched therapy.

\section{Results}

Of 4243 patients enrolled into the study, baseline documentation was available for 4200 of which 4196 fulfilled all inclusion criteria with a total of 431 (10.3\%) patients tested positive for EGFR Mut + tumors. The disposition of patients through the study has been previously reported [6]. Documented decision of first-line treatment was collected for 2946 patients 6 69\%; 2481 EGFR mutation-negative [Mut-; 58\%], 131 EGFR Mut unknown [3\%], and 334 EGFR Mut + [7\%]). The majority of patients $(84.9 \%)$ were treated in a hospital $(81.7 \%$ and $85.2 \%$ of patients with EGFR Mut + and EGFR Mut- disease, respectively): $59.8 \%$ inpatients, $27.8 \%$ outpatients, and $12.4 \%$ daytime care. A further $14.3 \%$ of patients were treated by an oncologist in private practice and $0.8 \%$ of patients were treated by a pneumologist. During this study, a greater proportion of patients with EGFR Mut- disease were treated as inpatients (63.7\%) compared with patients with EGFR Mut + disease (32.6\%).
The most common first-line treatments selected were carboplatin (45.5\%), cisplatin (33.9\%), and pemetrexed (28.2\%) (Table 1). TKIs/gefitinib were received as firstline therapy in $8.2 \% / 6.2 \%$ of all patients and $56.6 \% /$ $53.0 \%$ of patients with EGFR Mut + NSCLC ( $n=334)$. Combination chemotherapy, generally platinum-based, was received in $35.0 \%$ of patients with EGFR Mut + disease; $78.5 \%$ of $E G F R$ Mut- patients received combination chemotherapy and $12.9 \%$ received monochemotherapy. The most commonly used agents for patients with EGFR Mut- disease were carboplatin (48.5\%), cisplatin (36.2\%), and pemetrexed (30.4\%).

At follow-up, $58.8 \% / 55.0 \%$ of 320 patients with $E G F R$ Mut + NSCLC had received TKI/gefitinib therapy, 21.9\% were receiving combination chemotherapy, and 10.0\%/ 9.4\% had switched from combination chemotherapy to TKI/gefitinib therapy. First-line therapy was continued as maintenance in $71(22.2 \%)$ patients with EGFR Mut + NSCLC, mainly planned to be gefitinib (44 patients). There was an indication that older patients were more likely to receive TKIs than younger patients (odds ratio 1.05 , 95\% CI 1.01-1.09, $P=0.01$ ). Reasons why patients did not receive a TKI were not collected.

The most common second-line therapy choice among 122 patients with EGFR Mut + disease was TKI therapy followed by pemetrexed and platinum agents (Fig. 1). Nine patients received second-line treatment within a clinical study. Among the 26 patients receiving third- and subsequent-line treatment, pemetrexed was the most commonly used treatment, followed by a TKI (Fig. 1). Of the 320 EGFR Mut + patients with follow-up visits, 242/213 had documented TKI/gefitinib treatment (17 documented as planned TKI treatment). No TKI treatment was documented for 61 patients during the REASON study.

\section{Clinical outcomes}

Of the 334 patients with EGFR Mut + disease and documented first-line treatment, 320 were assessed for clinical outcome, of which 220/206 had received a TKI/ gefitinib during first-line treatment. The mean number of documented tumor evaluations per patient was 4.9 among those receiving first-line TKIs and 4.1 among those not receiving TKIs.

Among the 320 patients assessed for clinical outcome, the estimated median OS and PFS was 17.2 months and 9.1 months, respectively (Table 2). Among groups of patients analyzed, OS and PFS were longer in the following: female versus male; never smoker versus ever smoker (Table 2). Additionally, PFS was longer in the following: adenocarcinoma versus non-adenocarcinoma; TKI-sensitive versus TKI-insensitive EGFR mutations.

Of those patients who received a TKI/gefitinib before first disease progression, PFS was longer compared with those who did not receive a TKI (Fig. 2a and b). Analysis 
Table 1 First-line treatment decisions

\begin{tabular}{|c|c|c|c|c|}
\hline$n, \%$ & $\begin{array}{l}\text { EGFR Mut+ } \\
n=334\end{array}$ & $\begin{array}{l}\text { EGFR Mut- } \\
n=2481\end{array}$ & $\begin{array}{l}\text { EGFR Mx } \\
n=131\end{array}$ & $\begin{array}{l}\text { Total } \\
N=2946\end{array}$ \\
\hline \multicolumn{5}{|l|}{ Agent } \\
\hline Carboplatin & $74(22.2)$ & $1203(48.5)$ & $62(47.3)$ & $1339(45.5)$ \\
\hline Cisplatin & $60(18.0)$ & $897(36.2)$ & $43(32.8)$ & $1000(33.9)$ \\
\hline Pemetrexed & $39(11.7)$ & $754(30.4)$ & $38(29.0)$ & $831(28.2)$ \\
\hline Gemcitabine & $37(11.1)$ & $603(24.3)$ & $36(27.5)$ & $676(22.9)$ \\
\hline Vinorelbine & $43(12.9)$ & $586(23.6)$ & $41(31.3)$ & $670(22.7)$ \\
\hline Paclitaxel & $21(6.3)$ & $284(11.4)$ & $8(6.1)$ & $313(10.6)$ \\
\hline Gefitinib & $177(53.0)$ & $6(0.2)$ & 0 & $183(6.2)$ \\
\hline Bevacizumab & $18(5.4)$ & $142(5.7)$ & $1(0.8)$ & $161(5.5)$ \\
\hline Docetaxel & $3(0.9)$ & $97(3.9)$ & $1(0.8)$ & $101(3.4)$ \\
\hline Etoposide & $1(0.3)$ & $76(3.1)$ & $3(2.3)$ & $80(2.7)$ \\
\hline Erlotinib & $12(3.6)$ & $46(1.9)$ & $3(2.3)$ & $61(2.1)$ \\
\hline Other & 0 & $19(0.8)$ & $1(0.8)$ & $20(0.7)$ \\
\hline Cetuximab & 0 & $3(0.1)$ & 0 & $3(0.1)$ \\
\hline \multicolumn{5}{|l|}{ Type of treatment } \\
\hline Combination chemotherapy & $117(35.0)$ & $1947(78.5)$ & $103(78.6)$ & $2167(73.6)$ \\
\hline Monochemotherapy ${ }^{a}$ & $10(3.0)$ & $319(12.9)$ & $23(17.6)$ & $352(11.9)$ \\
\hline TKI & $189(56.6)$ & $49(2.0)$ & $3(2.3)$ & $241(8.2)$ \\
\hline Chemotherapy + bevacizumab and/or cetuximab & $18(5.4)$ & $141(5.7)$ & $1(0.8)$ & $160(5.4)$ \\
\hline Not classifiable & 0 & $19(0.8)$ & $1(0.8)$ & $20(0.7)$ \\
\hline Other & 0 & $6(0.2)$ & 0 & $6(0.2)$ \\
\hline
\end{tabular}

Patients with at least one specification of chemotherapy - multiple answers were permitted. Individual agents and treatment type ranked in order of decreasing use in the total population. Mut+, mutation-positive; Mut-, mutation-negative; Mx, mutation unknown/non-evaluable; TKl, tyrosine kinase inhibitor. ${ }^{a}$ Carboplatin,

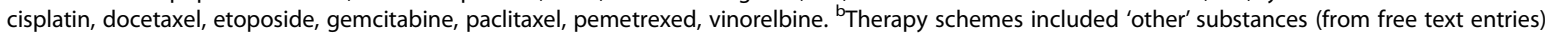

of OS showed no significant difference between these patient populations (Fig. 2c and d). However, longer OS was reported in those patients who ever received a TKI during their complete therapy course compared with those who never received a TKI: median OS 18.4 vs. 13.6 months; HR 0.53; $\log$-rank $p=0.003$ (Fig. 3a). A similar outcome was shown for those patients who ever received gefitinib compared with those who never received a TKI: median OS 18.1 vs. 13.6 months; HR 0.55; $\log$-rank $p=0.005$ (Fig. 3b).

RR was $50.9 \%$ overall (Table 2 ) and was higher in the following groups: female versus male; never smoker versus ever smoker; ever EGFR inhibitor versus never EGFR inhibitor; TKI-sensitive versus TKI-insensitive EGFR mutations.

\section{Pharmacoeconomic endpoints}

The three first-line treatment groups comprised chemotherapy $(n=90)$, TKI $(n=159)$, and switch to TKI $(n=31)$. Total cost of treatment was highest for the TKI group $(€ 46,443)$ and lowest for the chemotherapy group $(€ 27,182)$. For all three groups, cost of drug was the main expenditure. As a proportion of the total costs, drug costs were higher with TKI and switch therapy $(75.5 \%$ and $76.7 \%$, respectively) compared with chemotherapy (57.1\%). In terms of mean outpatient and inpatient costs, the chemotherapy group had the highest costs and the switch group the lowest (Additional file 1: Table S1).

The number of patients with a documented nursing auxiliary decreased during the course of observation in the chemotherapy group (13.7\% vs. $12.7 \%)$ and increased in the TKI and switch groups, by 5.4 percentage points (15.5\% vs. $20.9 \%)$ and 16.1 percentage points $(22.6 \%$ vs. $38.7 \%)$, respectively. The proportion of patients without a nursing auxiliary listed at the final visit was $62.6 \%, 60.8 \%$, and $51.6 \%$ for the TKI, chemotherapy, and switch groups, respectively.

The number of patients with an employment relationship decreased throughout the observation period in all three groups. The biggest changes were seen in the switch group (25.8\% to $3.2 \%)$, compared with the chemotherapy (28.4\% to $8.8 \%$ ) and TKI (18.2\% to $7.5 \%)$ groups. However, the chemotherapy group had a higher proportion of patients with an unknown employment relationship at the end of treatment (26.5\%) than the TKI (17.1\%) and switch (9.7\%) groups. At the last visit, the proportions of patients with full-time employment in the 


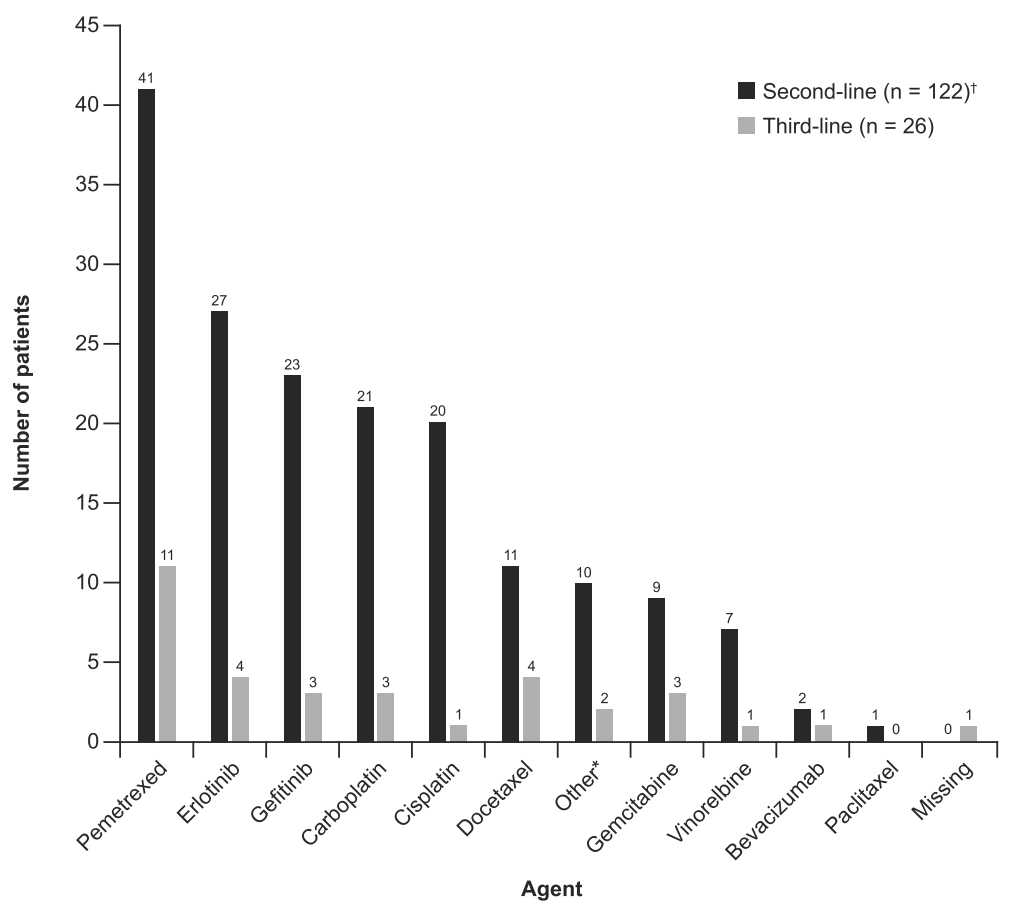

Fig. 1 Second- and third-line treatment in patients with EGFR Mut + NSCLC More than one agent could be reported. *Other = experimental ( $n=4 \mathrm{~s}$-line), afatinib ( $n=3 \mathrm{~s}$-line), experimental afatinib ( $n=2$ third-line), gefitinib/placebo ( $n=1 \mathrm{~s}$-line), trofosfamide $\left(n=1 \mathrm{~s}\right.$-line). ${ }^{\dagger}$ Data for patients receiving second-line treatment are a combination of planned treatment ( $n=63$ patients who did not consent to collection of data for second-line treatment) and actual treatment ( $n=59$ patients who consented to collection of data for second and subsequent lines of treatment). Mut+, mutation-positive; NSCLC, non-small-cell lung cancer

chemotherapy, TKI, and switch groups were $4.9 \%, 5.3 \%$, and $3.2 \%$, respectively.

\section{Safety}

Over half of the patients receiving gefitinib reported at least one $\mathrm{AE}$ (58.1\%), of which rash, diarrhea, and dry skin were the most common AEs (Table 3) and ADRs. A total of 20 grade 3-5 ADRs were reported, including two patients each with grade 3 rash, diarrhea, and nausea and two grade 4 reactions (diarrhea and thrombosis/thrombus/embolism).

Serious AEs were reported for 49 patients (22.1\%), the most frequent of which were cardiac ischemia/infarction and constitutional symptoms, other (2.3\%, each), followed by diarrhea and cystitis (1.8\%, each). Eight patients (3.6\%) had AEs leading to discontinuation of treatment with gefitinib, including diarrhea $(n=4)$ and nausea $(n=2)$. There were 11 deaths, only one of which was considered to be related to treatment with gefitinib (hemorrhage, pulmonary/upper respiratory - bronchopulmonary not otherwise specified).

\section{Discussion}

To date, the REASON study represents the largest dataset of information on EGFR mutations in Caucasian patients with NSCLC. In the REASON study, $10.3 \%$ of patients were tested positive for EGFR mutations, similar to the European population (12\%) in ASSESS, a large multicentre, non-interventional diagnostic study in patients with advanced NSCLC [7].

In patients with EGFR Mut + NSCLC who received a TKI (or gefitinib as their TKI) before first disease progression, PFS was prolonged by about three months compared with those who did not receive a TKI. The RR was higher in patients receiving first-line TKI than in those not receiving a TKI (53.2\% vs. $45.0 \%)$. Median OS was similar between those patients who received a TKI or gefitinib before first disease progression compared with those who did not receive a TKI. These outcomes for PFS, OS, and RR parallel those of clinical trials comparing TKIs with standard doublet chemotherapy regimens $[3,4,8]$.

A survival analysis of patients with EGFR Mut + NSCLC who ever received a TKI (or gefitinib as their TKI) during the course of their treatment revealed an increase in median OS of approximately five months compared with those who never received a TKI. However, when interpreting these data it should be considered that by virtue of surviving longer, patients may have received a greater number of treatments (including EGFR-TKIs) compared with those patients with poorer prognosis. This may have biased the REASON OS analysis in favor of those patients who ever received a TKI during their entire treatment course $(n=242)$ compared with those who never received a TKI $(n=61)$. 
Table 2 OS, PFS, and RR in patients with EGFR Mut + NSCLC

\begin{tabular}{|c|c|c|c|c|c|c|c|}
\hline & \multirow[t]{2}{*}{$\mathrm{n}$} & \multicolumn{2}{|c|}{ Overall survival } & \multicolumn{2}{|c|}{ Progression-free survival } & \multicolumn{2}{|c|}{ Response rate } \\
\hline & & $\begin{array}{l}\text { Median } \\
\text { (months) }\end{array}$ & $95 \% \mathrm{Cl}$ & $\begin{array}{l}\text { Median } \\
\text { (months) }\end{array}$ & $95 \% \mathrm{Cl}$ & $\mathrm{n}$ & $\%$ \\
\hline Overall & 320 & 17.2 & $15.1-19.8$ & 9.1 & $8.5-10.3$ & 163 & 50.9 \\
\hline \multicolumn{8}{|l|}{ Gender } \\
\hline Female & 200 & 20.4 & $17.2-23.8$ & 10.3 & $9.4-12.6$ & 110 & 55.0 \\
\hline \multirow[t]{2}{*}{ Male } & 120 & 12.2 & $9.6-17.0$ & 6.8 & $5.1-8.8$ & 53 & 44.2 \\
\hline & & \multicolumn{2}{|l|}{$P<0.001^{\mathrm{a}}$} & \multicolumn{2}{|l|}{$P<0.001^{\mathrm{a}}$} & & $P=0.078^{b}$ \\
\hline \multicolumn{8}{|l|}{ Histology } \\
\hline Adenocarcinoma & 286 & 17.0 & $15.1-19.5$ & 9.3 & $8.7-10.5$ & 148 & 51.7 \\
\hline \multirow[t]{2}{*}{ Non-adenocarcinoma } & \multirow[t]{2}{*}{33} & \multirow[t]{2}{*}{18.4} & \multirow[t]{2}{*}{$12.2-\mathrm{NA}$} & 6.9 & \multirow[t]{2}{*}{$5.1-21.3$} & \multirow[t]{2}{*}{15} & 45.5 \\
\hline & & & & $P=0.82^{\mathrm{a}}$ & & & $P=0.616^{\mathrm{b}}$ \\
\hline \multicolumn{8}{|l|}{ Smoking habit } \\
\hline Ever smoker & 168 & 15.1 & $13.6-18.1$ & 8.1 & $6.8-10.3$ & 79 & 47.0 \\
\hline \multirow[t]{2}{*}{ Never smoker } & \multirow[t]{2}{*}{150} & 20.4 & \multirow[t]{2}{*}{$17.0-26.5$} & 10.2 & \multirow[t]{2}{*}{$9.1-12.0$} & \multirow[t]{2}{*}{83} & 55.3 \\
\hline & & $P=0.014^{\mathrm{a}}$ & & $P=0.029^{\mathrm{a}}$ & & & $P=0.172^{b}$ \\
\hline \multicolumn{8}{|l|}{ First-line therapy } \\
\hline Ever EGFR inhibitor & 220 & 16.4 & $14.3-20.3$ & 9.6 & $8.8-11.1$ & 118 & 53.6 \\
\hline No EGFR inhibitor & 100 & 18.1 & $15.1-23.5$ & 8.7 & $6.3-11.2$ & 45 & 45.0 \\
\hline Ever gefitinib & 206 & 16.4 & $14.2-20.4$ & 9.6 & $8.6-10.9$ & 111 & 53.9 \\
\hline TKI & 188 & 17.4 & $14.7-20.4$ & 9.7 & $8.5-11.4$ & 100 & 53.2 \\
\hline Gefitinib & 176 & 17.4 & $14.7-20.4$ & 9.6 & $8.1-11.3$ & 94 & 53.4 \\
\hline Chemotherapy & 100 & 18.1 & $15.1-23.5$ & $8.7^{c}$ & $6.3-11.2$ & 45 & 45.0 \\
\hline Chemotherapy $\rightarrow$ TKI & 32 & 13.9 & 9.1-NA & 9.2 & $8.6-21.6$ & 18 & 56.3 \\
\hline Chemotherapy $\rightarrow$ gefitinib & 30 & 10.3 & $8.6-21.6$ & 13.8 & 8.6-NA & 17 & 56.7 \\
\hline TKI maintenance planned & 57 & 19.8 & 15.0-NA & 10.3 & $8.7-16.3$ & 38 & 66.7 \\
\hline No TKI maintenance planned & 263 & 16.4 & $14.2-19.1$ & 9.0 & $7.7-10.3$ & 125 & 47.5 \\
\hline TKI from start & 158 & 16.4 & $13.1-20.3$ & 9.7 & $7.6-11.4$ & & \\
\hline Change to TKI/planned TKI maintenance & 76 & 17.9 & 14.8-NA & 10.0 & $8.7-14.8$ & & \\
\hline No TKI & 86 & 18.0 & $14.2-22.5$ & 8.1 & $6.1-11.2$ & & \\
\hline TKI treatment ${ }^{\mathrm{d}, \mathrm{e}}$ & & & & & & & \\
\hline TKI from start & 188 & 17.4 & $14.7-20.4$ & 9.7 & $8.5-11.4$ & & \\
\hline TKI switch/planned maintenance & 46 & 17.0 & 10.0-NA & 10.0 & $8.6-21.4$ & & \\
\hline No TKI (first + maintenance) & 86 & 18.0 & $14.2-22.5$ & 8.1 & $6.1-11.2$ & & \\
\hline TKI documented & 229 & 17.9 & $15.0-20.5$ & 10.1 & $8.9-11.7$ & & \\
\hline Gefitinib documented & 206 & 17.4 & $14.8-20.4$ & 10.0 & $8.8-11.4$ & & \\
\hline Planned TKI documented & 12 & NA & NA & 8.7 & 3.6-NA & & \\
\hline No TKI documented & 79 & 15.4 & $13.8-22.5$ & 7.0 & $5.1-9.4$ & & \\
\hline TKI treatment ${ }^{\mathrm{e}, \mathrm{f}}$ & & & & & & & \\
\hline TKI documented & 242 & 18.4 & $16.3-21.8$ & & & & \\
\hline Gefitinib documented & 213 & 18.1 & $15.5-21.4$ & & & & \\
\hline Planned TKI documented & 17 & 17.0 & 10.0-NA & & & & \\
\hline No TKI documented & 61 & 13.6 & $9.3-15.4$ & & & & \\
\hline EGFR mutation & & & & & & & \\
\hline TKI-sensitive & 231 & 18.1 & $15.5-20.9$ & 10.2 & $9.1-11.7$ & 132 & 57.1 \\
\hline TKI-insensitive & 24 & 17.9 & 6.9-NA & 5.4 & $4.0-9.4$ & 8 & 33.3 \\
\hline & & & & & & & $P=0.044^{b}$ \\
\hline
\end{tabular}

OS, PFS, and RR by demographic and clinico-pathological characteristics, and therapy in patients with EGFR Mut + NSCLC. ${ }^{\mathrm{a}}$ Log-rank test. ${ }^{\mathrm{b}} \mathrm{Chi}-\mathrm{squared}$ test. ${ }^{\mathrm{C}}$ Includes two patients in whom the therapeutic agent was changed within first-line treatment but the new agent was not documented. ${ }^{\mathrm{d}}$ TKI until first documented tumor progression. ${ }^{e}$ Analysis not prespecified. Patients who ever received a TKI as part of their complete therapy course

$\mathrm{Cl}$, confidence interval; NA, not available; NSCLC, non-small-cell lung cancer; RR, response rate; OS, overall survival; PFS, progression-free survival; TKI, tyrosine kinase inhibitor 
a

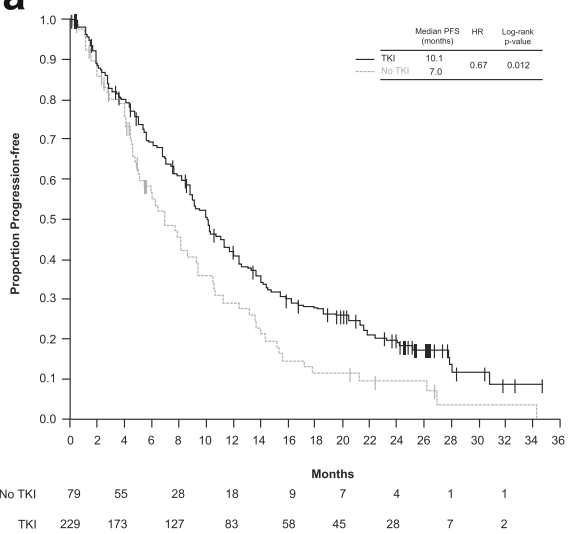

C

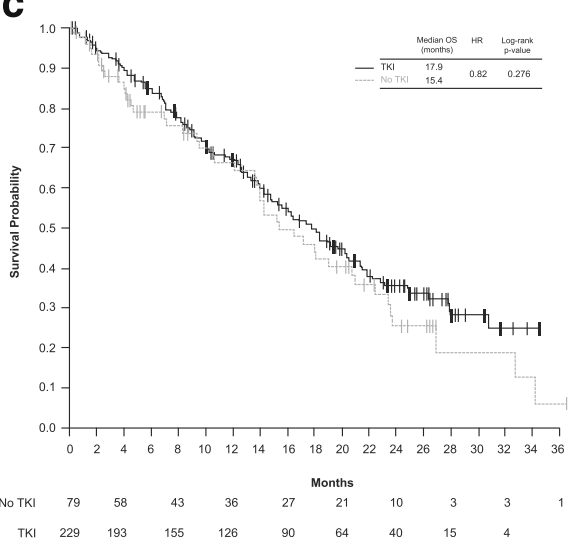

b

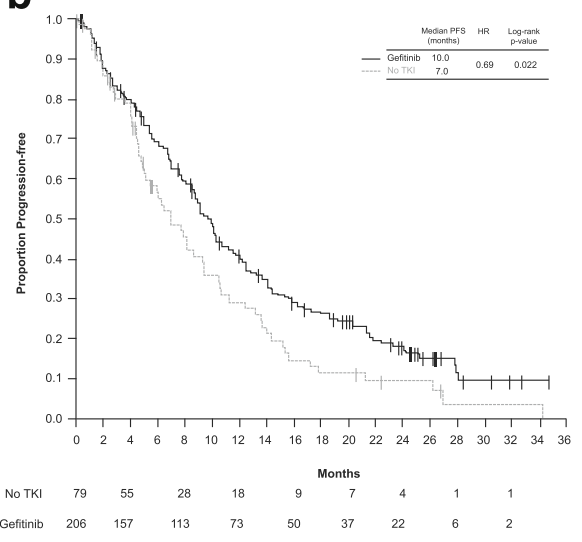

d

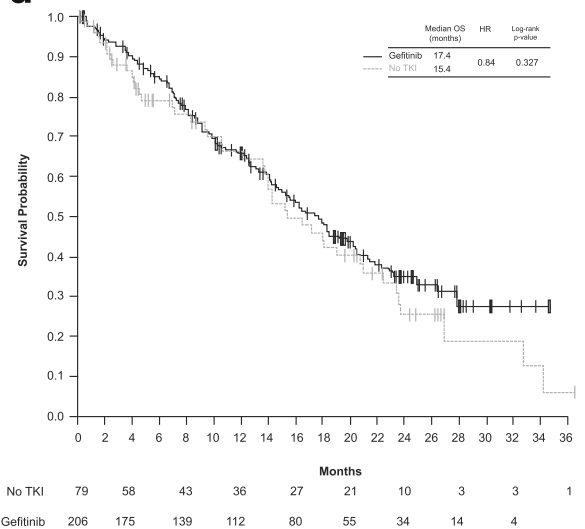

Fig. 2 KM estimates of PFS and OS: patients with EGFR Mut + advanced NSCLC by therapy Kaplan-Meier estimates of progression-free survival (a and $\mathbf{b}$ ) and overall survival (c and $\mathbf{d}$ ), of patients with EGFR Mut + advanced NSCLC who received either a TKI (a and $\mathbf{c}$ ) or gefitinib (b and $\mathbf{d}$ ) prior to first disease progression compared with those patients who did not receive a TKI prior to first disease progression. KM, Kaplan-Meier; Mut + , mutation-positive; NSCLC, non-small-cell lung cancer; TKI, tyrosine kinase inhibitor
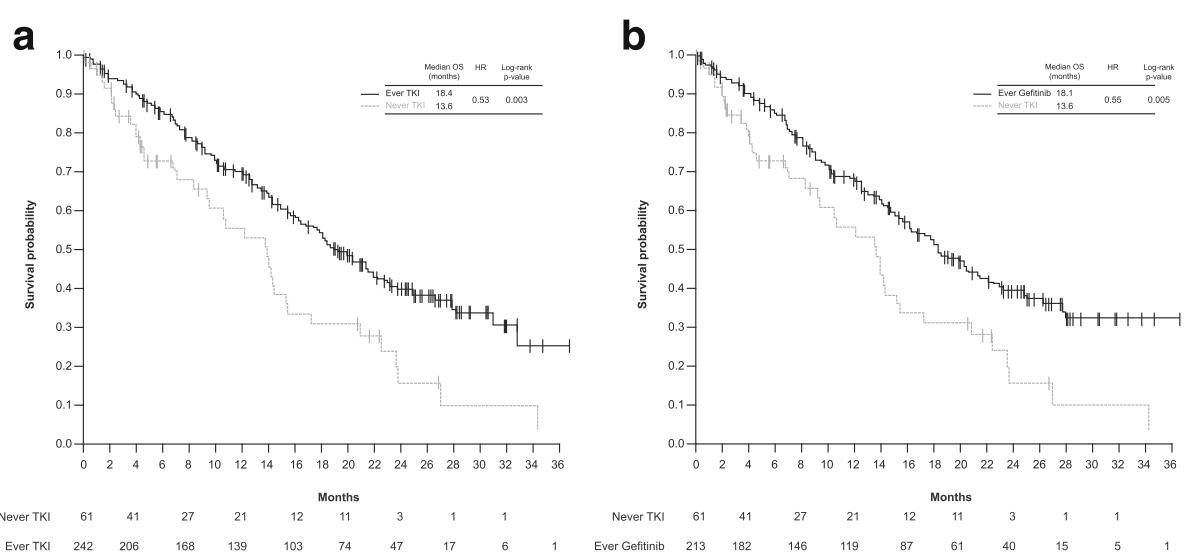

Fig. 3 KM estimates of OS: patients with EGFR Mut + advanced NSCLC who ever received a TKI Kaplan-Meier estimates of overall survival of patients with EGFR Mut + advanced NSCLC who ever received either a TKI (a) or gefitinib (b) during their entire course of treatment compared with those who did not receive a TKI. KM, Kaplan-Meier; Mut+, mutation-positive; NSCLC, non-small-cell lung cancer; TKI, tyrosine kinase inhibitor 
Table 3 AEs in patients with EGFR Mut + NSCLC treated with gefitinib ( $\geq 2 \%$ of patients)

\begin{tabular}{|c|c|c|}
\hline & $\begin{array}{l}n \\
(N=222)\end{array}$ & $\%$ \\
\hline All & 129 & 58.1 \\
\hline \multicolumn{3}{|l|}{ Dermatology/skin } \\
\hline Rash: acne/acneiform & 53 & 23.9 \\
\hline Dry skin & 24 & 10.8 \\
\hline Nail changes & 14 & 6.3 \\
\hline Pruritus/itching & 14 & 6.3 \\
\hline Dermatology/skin - other & 11 & 5.0 \\
\hline Hair loss/alopecia & 9 & 4.1 \\
\hline Rash/desquamation & 8 & 3.6 \\
\hline \multicolumn{3}{|l|}{ Gastrointestinal } \\
\hline Diarrhea & 40 & 18.0 \\
\hline Nausea & 17 & 7.7 \\
\hline Vomiting & 8 & 3.6 \\
\hline \multicolumn{3}{|l|}{ Cardiac general } \\
\hline Cardiac ischemia/infarction & 5 & 2.3 \\
\hline \multicolumn{3}{|l|}{ Constitutional symptoms } \\
\hline Constitutional symptoms - other & 6 & 2.7 \\
\hline Fatigue (asthenia, lethargy, malaise) & 5 & 2.3 \\
\hline \multicolumn{3}{|l|}{ Ocular/visual } \\
\hline Other & 6 & 2.7 \\
\hline \multicolumn{3}{|l|}{ Hemorrhage/bleeding } \\
\hline Hemorrhage, pulmonary/upper respiratory - nose & 5 & 2.3 \\
\hline \multicolumn{3}{|l|}{ Neurology } \\
\hline Neuropathy: sensory & 5 & 2.3 \\
\hline \multicolumn{3}{|l|}{ Pulmonary/upper respiratory } \\
\hline Dyspnea (shortness of breath) & 5 & 2.3 \\
\hline \multicolumn{3}{|l|}{ Renal/genitourinary } \\
\hline Cystitis $^{a}$ & 4 & 1.8 \\
\hline
\end{tabular}

Adverse events by CTC symptoms related to gefitinib and serious adverse events related and not related to gefitinib. AE, adverse event; CTC, Common Toxicity Criteria; NSCLC, non-small-cell lung cancer. ${ }^{~ I n c l u d e s ~ o n e ~ p a t i e n t ~ i n ~}$ whom cystitis was not related to gefitinib and was not serious

Previous real world studies suggested that patients with EGFR Mut + disease who receive targeted therapy survive longer $[9,10]$. In contrast the EPICLIN-lung study did not show any benefit, most likely because TKIs were often used without selection for EGFR mutation [11]. To date no significant differences in PFS between gefitinib and erlotinib have been reported in real world studies [12, 13].

In the REASON study, first-line treatments for all patients commonly included platinum agents and pemetrexed, similar to the findings from MUTACT (a French observational study on the management of patients with NSCLC adenocarcinoma) [14]. Altogether,
$6.2 \%$ of patients in the REASON study received gefitinib as first-line treatment, fewer than reported in the MUTACT study (23\%). There were also fewer patients with EGFR Mut + NSCLC receiving a TKI firstline in the REASON study (56.6\%) compared with the MUTACT study (76\%). As previously reported, this possibly reflects patients with acute symptoms initiating first-line chemotherapy while waiting for EGFR mutation test results and who subsequently switch to an EGFR-TKI once a positive mutation test was confirmed [6]. The proportion of patients with EGFR Mut + NSCLC who ever received a TKI during their entire treatment course was $80 \%$ (242/303 patients). This is broadly in line with an Asian retrospective cohort study of patients with advanced NSCLC, in which $88 \%$ of the patients with EGFR Mut + NSCLC received a TKI at some point in their treatment (first, second-, or third-line) [15]. The majority of patients in REASON with EGFR Mut + NSCLC who received an EGFR-TKI first-line were prescribed gefitinib over erlotinib; this could be explained by the regulatory status of the EGFR-TKIs at the time of the REASON study. Gefitinib was approved for use in patients with locally advanced or metastatic NSCLC with activating mutations of EGFR-TK in July 2009, whereas erlotinib was approved as a first-line monotherapy in the same group of patients in September 2011, 2 years after the start of REASON [16, 17]. Pemetrexed was the most commonly used second- and third-line treatment for patients with EGFR Mut + NSCLC, followed by erlotinib and gefitinib.

The cost of treating patients during first-line therapy until progression was $40 \%$ lower in the chemotherapy group than in the TKI group. For all three groups, drug costs were the main expense, followed by inpatient costs. Drug costs for chemotherapy were around half compared with the TKI and switch groups. However, the highest mean outpatient and inpatient costs were documented for chemotherapy patients. It should be noted that the AE profile of gefitinib in the REASON study was consistent with that described in the Summary of Product Characteristics [18]. At the end of the observation period, more patients in the TKI group did not have a nursing auxiliary listed compared with the chemotherapy group (62.6\% vs. $51.6 \%$ ). Taken together, these data suggest EGFR-TKIs as first-line treatment in patients with EGFR Mut + NSCLC results in fewer medical interventions than with chemotherapy. This is supported by a study on the impact of targeted treatment on direct medical costs of patients with advanced NSCLC, which showed targeted agents for patients with EGFR Mut + NSCLC lowered the mean monthly medical costs by prolonging survival and diminishing the use of other medical resources [19]. 
The numbers of patients with employment relationships at the end of the observation period were low in all treatment groups. They were particularly low for switch patients $(3.2 \%$ vs. $8.8 \%$ for chemotherapy and $7.5 \%$ for TKI therapy). However, the larger number of patients with an unknown employment relationship at the end of observation in the chemotherapy group compared with the other two groups challenges the interpretation of these data.

\section{Conclusions}

Findings from the REASON study secondary endpoints provide a valuable insight into current treatment patterns, clinical outcomes and resource use in patients with EGFR Mut + NSCLC in Germany. In summary, RR, PFS and OS with first-line EGFR-TKI treatment for patients with EGFR Mut + advanced NSCLC are in line with expectations based on previous clinical trials. OS analysis across the entire treatment course reveals a benefit in those patients who ever received an EGFRTKI vs those who did not, which is in line with other real-world evidence [10]. The cost of first-line EGFRTKI treatment is more expensive than chemotherapy; however, the highest mean outpatient and inpatient costs were documented for chemotherapy patients, and at the end of the observation period, more patients in the TKI group did not have a nursing auxiliary listed compared with the chemotherapy group.

\section{Additional file}

Additional file 1: Table S1. Treatment costs according to type of firstline treatment received by patients with EGFR Mut + NSCLC. (DOCX $15 \mathrm{~kb}$ )

\section{Abbreviations}

ADR: Adverse drug reaction; AE: Adverse event; EGFR: Epidermal growth factor receptor; NSCLC: Non-small cell lung cancer; OS: Overall survival; PFS: Progressionfree survival; REASON: Registry for the epidemiological and scientific evaluation of EGFR mutation status in patients with newly diagnosed locally advanced or metastatic NSCLC; RECIST: Response evaluation criteria in solid tumours; RR: Response rate; TKl: Tyrosine kinase inhibitor

\section{Acknowledgements}

Medical writing services were provided by Tom Hudson of iMed Comms and were funded by AstraZeneca. The authors thank the patients and investigators who participated in this study.

\section{Funding}

The study was funded by AstraZeneca, Germany. WS received research funding from Roche and Lilly. WE received research funding from AstraZeneca and Eli Lilly. MT received financial support for this study from AstraZeneca. PS, UZ, LM, MT and MD report no funding.

\section{Availability of data and materials}

The data that support the findings of this study can be requested from the study sponsor, AstraZeneca, via the Data Request Portal (https:// astrazenecagroup-dt.pharmacm.com/DT/Home). The request will be evaluated and reviewed by AstraZeneca on a case-by-case basis.

\section{Authors' contributions}

WS, PS, WE and LM contributed to the conception and design of this study. LM was responsible for development and methodology. WE, UZ and MT acquired data. PS, WE, MD, UZ, LM and MT analysed and interpreted data. WE, MD, UZ, LM and MT were major contributors to writing and revision of the manuscript. UZ supervised the study. All authors read and approved the final manuscript.

\section{Ethics approval and consent to participate}

All procedures performed in studies involving human participants were in accordance with the ethical standards of the institutional and/or national research committee and with the 1964 Helsinki declaration and its later amendments or comparable ethical standards.

The opinion from the Ethics Committee of the coordinating investigator (Ärztekammer Sachsen-Anhalt) was sought for the final study protocol, including the final version of the Informed Consent Form. Notifications were sent to the Ethics Committees of all involved investigators. An Ethics Committee opinion was also sought for any amendment to the protocol in accordance with local requirements.

All patients provided written, informed consent

This article does not contain any studies with animals performed by any of the authors.

\section{Consent for publication}

Not applicable

\section{Competing interests}

WS reports honoraria from, Roche, Lilly and Boehringer Ingelheim, consulting or advisory roles with Roche, Lilly and Boehringer Ingelheim and travel, accommodation or expenses from Boehringer Ingelheim. PS reports honoraria from AstraZeneca, Novartis, Roche, Amgen and Pfizer and consulting or advisory roles with AstraZeneca, Novartis, Amgen and Pfizer. WE reports honoraria from AstraZeneca, Eli Lilly, Boehringer Ingelheim, Pfizer, Novartis, Roche, Merck, BristolMyers Squibb, Amgen, GlaxoSmithKline, Astellas, Bayer, Teva, Merck Serono, Daichi Sankyo and Hexal and consulting or advisory roles with AstraZeneca, Eli Lilly, Boehringer Ingelheim, Novartis, Pfizer, Roche, Merck, Bristol-Myers Squibb, Astellas, Bayer, Teva and Daichi Sankyo. UZ is an employee of iOMEDICO AG. LM is an employee of AstraZeneca. MT reports honoraria from AstraZeneca, Roche, BristolMyers Squibb, MSD, Lilly, Novartis and Pfizer and consulting or advisory roles with AstraZeneca, Bristol-Myers Squibb, MSD, Lilly, Novartis and Roche. MD reports no potential conflicts of interest.

\section{Publisher's Note}

Springer Nature remains neutral with regard to jurisdictional claims in published maps and institutional affiliations.

\section{Author details}

'Krankenhaus Martha-Maria Halle-Doelau gGmbH, Klinik für Innere Medizin II, Roentgenstr, 106120 Halle, Germany. ${ }^{2}$ Pathologisches Institut, Universitätklinik Heidelberg, Heidelberg, Germany. ${ }^{3}$ Department of Medical Oncology, West German Tumor Centre, University Hospital Essen, Rurhlandlkinik, University Duisburg-Essen, Essen, Germany. ${ }^{4}$ Pathologisches Institut Humboldt, Universität Berlin, Berlin, Germany. ${ }^{5} \mathrm{iOMEDICO}$ AG, Freiburg, Germany. ${ }^{6}$ Medical Affairs, AstraZeneca, Wedel, Germany. ${ }^{7}$ Internistische Onkologie der Thoraxtumoren, Thoraxklinik im Universitätsklinikum Heidelberg, Translational Lung Research Center Heidelberg (TLRC-H), Member of the German Center for Lung Research, Heidelberg, Germany.

Received: 29 November 2016 Accepted: 23 January 2018 Published online: 05 February 2018

References

1. Novello S, Barlesi F, Califano R, et al. Metastatic non-small-cell lung cancer: ESMO clinical practice guidelines for diagnosis, treatment and follow-up. Ann Oncol. 2016;27(suppl 5):v1-v27.

2. Li T, Kung HJ, Mack PC, Gandara DR. Genotyping and genomic profiling of non-small-cell lung cancer: implications for current and future therapies. J Clin Oncol. 2013:31:1039-49.

3. Ferlay J, Steliarova-Foucher E, Lortet-Tieulent J, et al. Cancer incidence and mortality patterns in Europe: estimates for 40 countries in 2012. Eur J Cancer. 2013;49:1374-403. 
4. Maemondo M, Inoue A, Kobayashi K, et al. Gefitinib or chemotherapy for nonsmall-cell lung cancer with mutated EGFR. N Engl J Med. 2010;362:2380-8.

5. German Cancer Guidelines. Lungenkarzinom, nicht-kleinzellig (NSCLC). Onkopedia 2016. https://www.onkopedia.com/de/onkopedia/quidelines/ lungenkarzinom-nicht-kleinzellig-nsclc/@@view/html/index.html. Accessed 1 Apr 2016.

6. Schuette W, Schirmacher P, Eberhardt WE, et al. EGFR mutation status and first-line treatment in patients with stage III/IV non-small cell lung cancer in Germany: an observational study. Cancer Epidemiol Biomark Prev. 2015;24: 1254-61.

7. Reck M, Hagiwara K, Han B, et al. ctDNA determination of EGFR mutation status in European and Japanese patients with advanced NSCLC: the ASSESS study. J Thorac Oncol. 2016;11(10):1682-9.

8. Mok TS, Wu YL, Thongprasert S, et al. Gefitinib or carboplatin-paclitaxel in pulmonary adenocarcinoma. N Engl J Med. 2009:361:947-57.

9. Kris MG, Johnson B, Berry $L$, et al. Treatment with therapies matched to oncogenic drivers improves survival in patients with lung cancers: results from the lung cancer mutation consortium (LCMC). Sydney, Australia: Presented at the WCLC, 2013. Abstract PL03.07. p. 27-31. http://pic1.cmt. com.cn/newspic/files/\%E4\%BC\%9A\%E8\%AE\%AE\%E6\%97\%A5\%E7\%A8\%8B. pdf. Accessed 29 Jan 2018.

10. Clinical Lung Cancer Genome Project (CLCGP), Network Genomic Medicine (NGM). A genomics-based classification of human lung tumors. Sci Transl Med. 2013;5(209):ra153.

11. Carrato A, Vergnenegre A, Thomas M, McBride K, Medina J, Cruciani G. Clinical management patterns and treatment outcomes in patients with non-small cell lung cancer (NSCLC) across Europe: EPICLIN-lung study. Curr Med Res Opin. 2014;30:447-61

12. Knetki-Wroblewska M, Kowalski DM, Zajda K, et al. Gefitinib in patients with advanced non-small-cell lung cancer. Pneumonol Alergol Pol. 2012:80:439-49.

13. Lu RL, Hu CP, Yang HP, Li YY, Gu QH, Wu L. Biological characteristics and epidermal growth factor receptor tyrosine kinase inhibitors efficacy of EGFR mutation and its subtypes in lung adenocarcinoma. Pathol Oncol Res. 2014; 20:445-51.

14. Souquet P-J, Fournel P, Locher C, et al. MUTACT: an observational study of EGFR mutation status and management of patients with non-small cell lung cancer (NSCLC) adenocarcinoma. Ann Oncol. 2012;23(Suppl 9):ix400-46. (Abstract 1270P)

15. Sharma SV, Bell DW, Settleman J, Haber DA. Epidermal growth factor receptor mutations in lung cancer. Nat Rev Cancer. 2007:7:169-81.

16. AstraZeneca. IRESSA (Gefitinib) receives marketing authorisation for the treatment of non-small cell lung cancer in Europe [press release]. AstraZeneca UK Ltd. 2009. https://www.astrazeneca.com/media-centre/ press-releases/2009/IRESSA-Gefitinib-Receives-Marketing-Authorisation01072009.html\#. Accessed 1 Apr 2016.

17. Roche. Roche's Tarceva receives European approval for first-line use in a genetically distinct type of lung cancer [press release]. F. Hoffmann-La Roche Ltd. 2011. https://www.roche.com/media/store/releases/med-cor2011-09-01.htm. Accessed 1 Apr 2016.

18. Eberhardt W, Thomas M, Graf von der Schulenberg J-M, et al. EGFR mutation testing and first-line treatment of patients with advanced NSCLC and positive EGFR mutation status: results from a German registry. Eur J Cancer. 2011:47:5636

19. Lee JK, Kim DW, Keam B, et al. The impact of molecularly targeted treatment on direct medical costs in patients with advanced non-small cell lung cancer. Cancer Res Treat. 2015:47:182-8.

\section{Submit your next manuscript to BioMed Central and we will help you at every step:}

- We accept pre-submission inquiries

- Our selector tool helps you to find the most relevant journal

- We provide round the clock customer support

- Convenient online submission

- Thorough peer review

- Inclusion in PubMed and all major indexing services

- Maximum visibility for your research

Submit your manuscript at www.biomedcentral.com/submit

) Biomed Central 